\title{
Looking for the immunological "time window" for the surgical intervention in patients with polytrauma
}

\author{
WOJCIECH MARKS ${ }^{I^{*}}$, KATARZYNA GOEĄBEK-DROPIEWSKA ${ }^{I^{*}}$, EWA BRYL ${ }^{2}$,

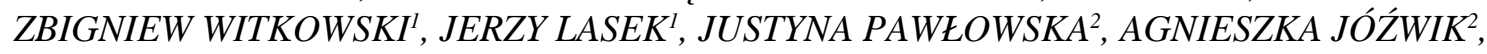 \\ JAN WIERUSZEWSKI ${ }^{3}$, ROBERT DUDEK ${ }^{3}$, JACEK WITKOWSKI \\ ${ }^{1}$ Department of Trauma Surgery, Medical University of Gdańsk, Poland \\ ${ }^{2}$ Department of Pathophysiology, Medical University of Gdańsk, Poland \\ ${ }^{3}$ Medical University of Gdańsk, Poland \\ * These two authors were equally participating in the experimental planning and performance of this work.
}

\begin{abstract}
Background: Polytrauma patients with a severity score over 16 points are at a risk of development of immunologically derived complications (infection, "2nd-hit injuries"). The aim of this study is to evaluate the phagocytic arm of the immune system in patients with polytrauma during a period of time, which is critical for therapeutic decisions. The phagocytic arm is analyzed to optimize the timing of definitive surgery.

Material and methods: The study group consisted of 7 men and 5 women from 20 to 84 years of age who had sustained a polytrauma as a result of mechanical factors. Polytrauma was severe (over 16 points ISS - Injury Severity Score) in all of the cases. All patients were studied on the $3^{\text {rd }}$ and $6^{\text {th }}$ day after trauma. Nine CNS-injured patients (isolated injury) and eleven healthy age-and sex-matched volunteers served as controls.

Results: A statistically significant and higher individual cellular phagocytic activity (number of bacteria per cell) was observed in polytrauma patients on the $3^{\text {rd }}$ day when compared to CNS-injured patients. The percentages of granulocytes showing phagocytosis in polytrauma patients on the $3^{\text {rd }}$ and $6^{\text {th }}$ day were significantly lower when compared to CNS-injured patients and healthy controls.

The percentages of granulocytes showing enzymatic activity in polytrauma patients on the $3^{\text {rd }}$ and $6^{\text {th }}$ day were significantly lower comparing to CNS-injured patients and healthy controls. Statistically significant and higher enzymatic activity of granulocytes was observed on the $3^{\text {rd }}$ and $6^{\text {th }}$ day in polytrauma patients when compared to healthy controls.

Conclusions: A significant deficiency of the phagocytic arm was observed during a period of time, which is critical for definitive surgical interventions in polytrauma patients. The phagocytic arm should be analyzed to optimize the timing of definitive surgery.
\end{abstract}

Key words: polytrauma, immunology, CNS injury, timing of definitive surgery, flow cytometry, phagocytosis, and oxygen burst.

(Centr Eur J Immunol 2013; 38 (4): 530-536)

\section{Introduction}

Severe trauma is associated with an early over-activation of an innate immune response followed by immunosuppression $[1,2]$. Trauma induces both immunosuppressive and catabolic states that are unable to clear infection [24]. It is reported that the patients with a severe CNS injury also showed a profound acute phase systemic response [3]. The next phase of this response is CNS injury-induced immunodepression (CIDS) [4]. Up until now we do not know the exact time line of these two phases. Over a period of months following the injury the immune response returns to normal [5]. This might suggest a decreased host defense in that period of time. The decreased host defense is also an essential component to trigger the "second-hit" mechanism. Despite the observed immunological deficiency, the period of time between the $3^{\text {rd }}$ and $6^{\text {th }}$ days is also critical as a window of opportunity for definitive surgery [6].

The aim of this study is to characterize the changing functional behavior of the peripheral blood granulocytes on the $3^{\text {rd }}$ day and the $6^{\text {th }}$ day post polytrauma injury, using

Correspondence: Wojciech Marks, Department of Trauma Surgery, Medical University of Gdańsk, Dębinki 7, 80-952 Gdańsk, Poland, tel./fax +485834924 02, e-mail: w_marks@wp.pl 
flow cytometric analysis of phagocytosis and oxidative burst activities.

\section{Material and methods}

\section{Patient population}

Patients admitted to the Department of Trauma Surgery at the Medical University of Gdańsk, following accidental trauma, were included in the prospective clinical study, which started on 1 November 2005 and lasted until 1 May 2009.

The study group consisted of 12 polytrauma injured patients ( 7 men and 5 women) who were 20 to 84 years of age and sustained a severe polytrauma injury (ISS higher than 16 pts), and were admitted to the Department of Trauma Surgery or Intensive Care Unit (ICU) at the Medical University of Gdańsk hospital. All polytrauma injured patients sustained a moderate or severe CNS injury and a moderate or severe injury of another body region (thorax, abdomen or extremities). All major surgical procedures were performed before the $3^{\text {rd }}$ day of hospitalization. The injuries were caused by mechanical trauma in all cases.

The first control group consisted of 9 brain injured patients ( 7 men and 2 women) who were 18 to 79 years of age and sustained a moderate or severe CNS injury (GCS not higher than $11 \mathrm{pts}$ ), and who were admitted to the Department of Trauma Surgery or Intensive Care Unit (ICU) at the Medical University of Gdańsk hospital. The injuries were caused by mechanical trauma in all cases. Patients with coexisting trauma were excluded.

All brain injured patients were clinically evaluated on admission and scored 11 points or less on the GCS (moderate or severe brain damage). Seven patients were artificially ventilated, five patients had an operation on the day of admission (craniotomy, hematoma evacuation), all patients received parenteral fluids. The nutritional condition of all patients was appropriate and has been maintained by administration of adequate parenteral solutions.

The second control group consisted of 11 qualified healthy subjects ( 3 women, 8 men). They were 17 to 82 years of age and with no history of polytrauma or craniocerebral trauma and no known immunodeficiencies.

The Local Ethics Committee of the Medical University of Gdańsk (MUG) approved the project.

\section{Methods}

We have measured four parameters: the function of granulocytes - the intensity of oxygen reactions; the number of bacteria per cell; the phagocytic activity - the number of granulocytes that shows phagocytosis; the number of granulocytes that have produced reactive oxygen metabolites.

All four parameters were measured on the $3^{\text {rd }}$ and $6^{\text {th }}$ day after trauma.
The intensity of oxidative burst and the number of granulocytes that have produced reactive oxygen metabolites were determined by the Bursttest ${ }^{\mathrm{TM}}$ (Orpagen) and then measured by means of flow cytometry. The number of bacteria per cell and the percentage of granulocytes that shows phagocytosis were measured by the Phagotest ${ }^{\mathrm{TM}}$ (Orpagen) and also by means of flow cytometry.

We have incubated and heparinized this blood sample with the labeled bacteria (E. coli) at $37^{\circ} \mathrm{C}$ to evaluate the phagocytic activity of the granulocytes in the peripheral blood sample. At the same time the control sample has remained on ice at $0^{\circ} \mathrm{C}$. Both placing it on ice and adding a quenching solution stopped the reaction in the first sample. The quenching solution allows us to differentiate the phagocytizing and non-phagocytizing cells. Then after two washing steps we have removed the remaining erythrocytes. Afterwards, the DNA staining solution was added to exclude artifacts. At the end, the acquisition gate was set at the proper fluorescence histogram to exclude the bacteria and platelet aggregates.

To assess the oxygen-dependent processes (the percentage of cells that have produced reactive oxygen metabolites and their mean fluorescence activity) we have tested the patients' and control group's blood samples by means of the Bursttest ${ }^{\mathrm{TM}}$. The heparinized blood samples were incubated with the following stimuli: E. coli bacteria, PMA and fMLP. The fourth sample without stimulus served as a control. As a result of stimulation, granulocytes produced oxygen metabolites, which was observed by oxidation of dihydrorhodamine DHR 123. The process was stopped by addition of the lysing solution. Then, the DNA staining solution was added. The number (percentage) of cells producing oxygen radical as well as their mean enzymatic activity was assessed by means of the flow cytometry.

Fluorescence data from both tests were obtained using the FACScan (Becton Dickinson, USA) cytometer. The minimum number of 10,000-15,000 of leukocytes per sample was collected.

The percentage of cells having performed phagocytosis (granulocytes) is analyzed as well as their mean fluorescence intensity (number of ingested bacteria). For that purpose, the relevant leukocyte cluster is gated in the software program in the scatter diagram (lin FSC vs. lin SSC) and its green fluorescence histogram (FL1) is analyzed (see Fig. 1A, 1B).

The Friedman ANOVA nonparametric test measures analysis of variance by ranks. This test was performed to evaluate the differences between compared groups. The U Mann-Whitney test was used in the analysis of data.

\section{Results}

A statistically significantly higher individual cellular phagocytic activity (number of bacteria per cell) was observed in polytrauma patients on the $3^{\text {rd }}$ day comparing to CNS-injured patients (Fig. 2). 


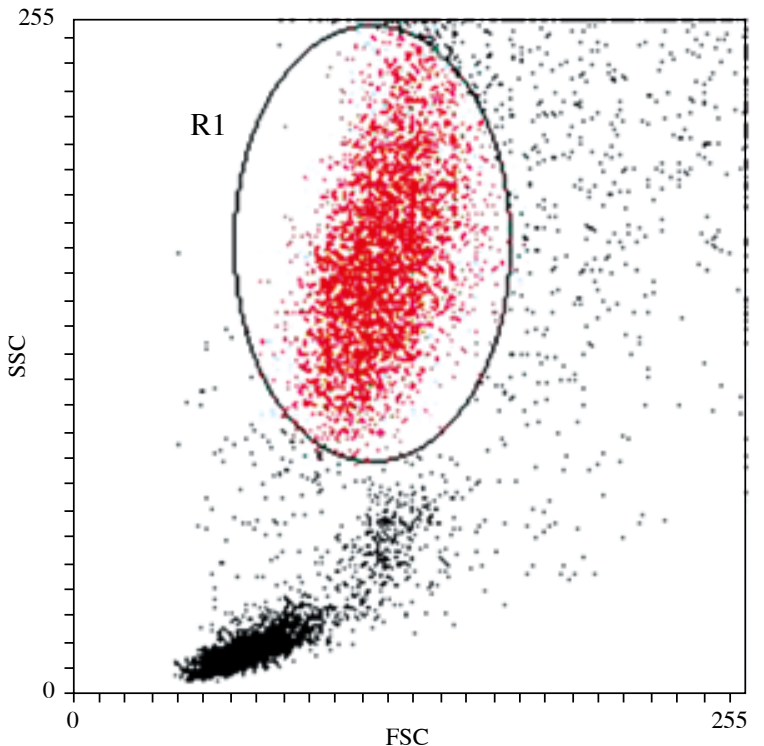

Fig. 1A. Typical dot plots FSC/SSC histograms of the phagocytosis test (incubation time of $10 \mathrm{~min}$ at $37^{\circ} \mathrm{C}$ ). Gate set on granulocytes

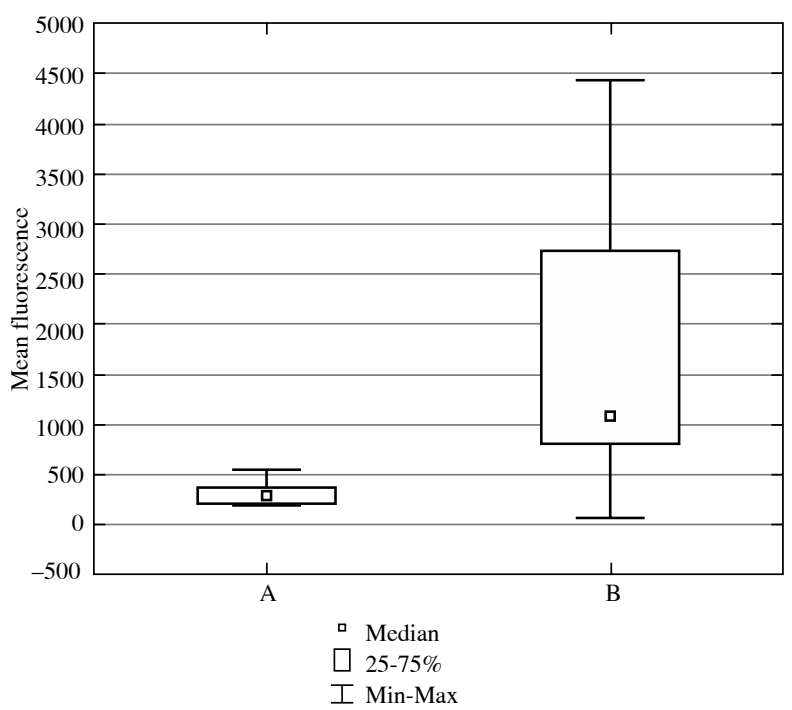

Fig. 2. Phagocytic activity (number of bacteria per cell) in polytrauma patients on the $3^{\text {rd }}$ day comparing to CNS-injured patients $(p<0.05)$

The percentages of granulocytes showing phagocytosis in polytrauma patients on the $3^{\text {rd }}$ and $6^{\text {th }}$ day were significantly lower comparing to CNS-injured patients and healthy controls (Fig. 3A-D).

The percentages of granulocytes that showed enzymatic activity in polytrauma patients on the $3^{\text {rd }}$ and $6^{\text {th }}$ day were significantly lower when compared to CNS-injured patients and healthy controls (Fig. 4A-D).

\section{B}

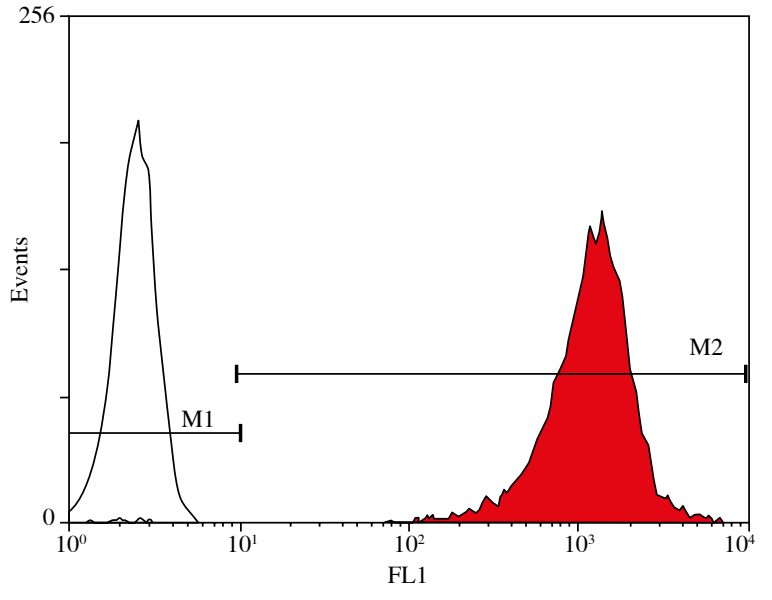

Fig. 1B. Typical FL1 histograms of the phagocytosis test (incubation time of $10 \mathrm{~min}$ at $37^{\circ} \mathrm{C}$ ). Gate set on granulocytes

A statistically significantly higher enzymatic activity of granulocytes was observed on the $3^{\text {rd }}$ and $6^{\text {th }}$ day in polytrauma patients when compared to healthy controls (Fig. 5A, B).

There were no significant differences in the phagocytic activity on the $6^{\text {th }}$ day when we compared polytrauma patients to the CNS-injured group and no significant differences in enzymatic activity on the $3^{\text {rd }}$ and $6^{\text {th }}$ day when polytrauma patients were compared to the CNS-injured patients.

There were no significant differences in the phagocytic activity on the $3^{\text {rd }}$ and $6^{\text {th }}$ day when polytrauma patients were compared to healthy controls.

There was no statistically significant tendency in all observed parameters on the $3^{\text {rd }}$ day when compared to the $6^{\text {th }}$ day after trauma in both polytrauma and CNS-injured group.

\section{Discussion}

According to the German Data Registry database, 2/3 of polytrauma patients also sustained relevant, severe CNS injuries. In these patients, 1/3 of CNS injuries were combined with injuries of the extremities [7].

CNS injury has been reported to induce various immunological abnormalities, including peripheral granulocytes hyperactivation under the influence of proinflammatory mediators in the first $12 \mathrm{~h}$ post trauma [8]. Thereafter, they enter a post excitatory phase wherein they become reduced in number and functionally deficient, placing the patient at a high risk of the "second-hit" injury [8].

The process of pathogen elimination by granulocytes constitutes an essential, primary event in the host defense against bacterial or fungal infections. This process can be separated into several major stages: chemotaxis (migration of granulocytes to inflammatory sites), attachment of par- 
A

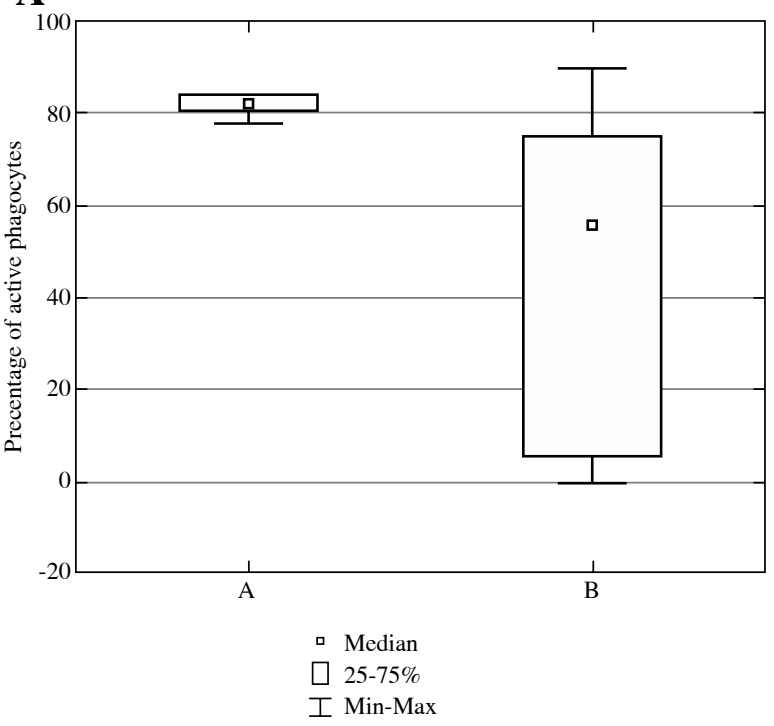

Fig. 3A. The percentage of granulocytes that showed phagocytosis in polytrauma patients on the $3^{\text {rd }}$ day when compared to the CNS-injured patients $(p<0.05)$

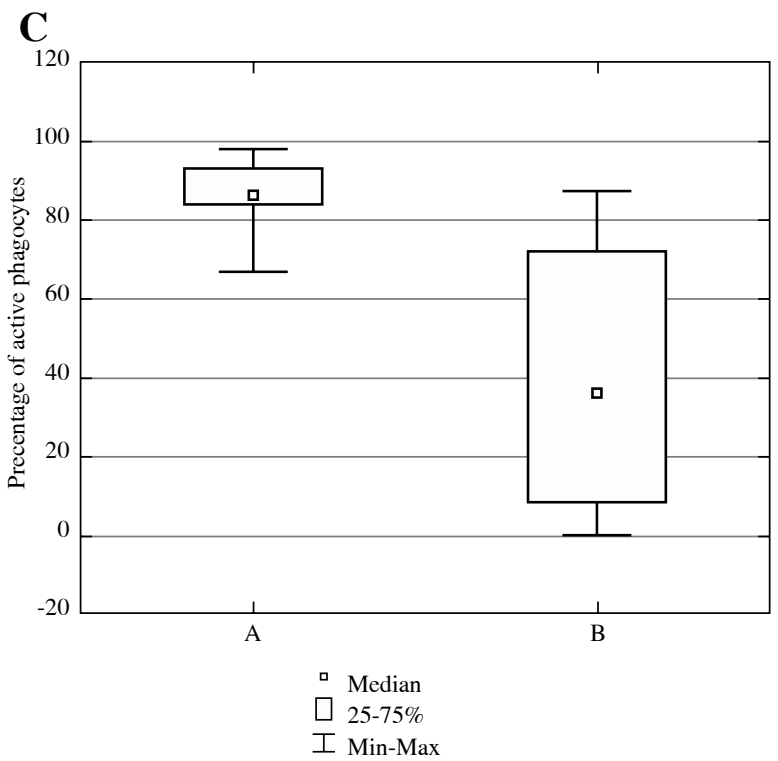

Fig. 3C. The percentage of granulocytes that showed phagocytosis in polytrauma patients on the $6^{\text {th }}$ day when compared to the CNS-injured patients $(p<0.05)$

ticles to the cell surface of granulocytes, ingestion (phagocytosis) and intracellular killing by oxygen-dependent (oxidative burst) and oxygen-independent mechanisms [9] .

The activity of the phagocytic arm of the immune system soon after the injury seems to be crucial for the appearance of infection and second-hit complications [10-13].

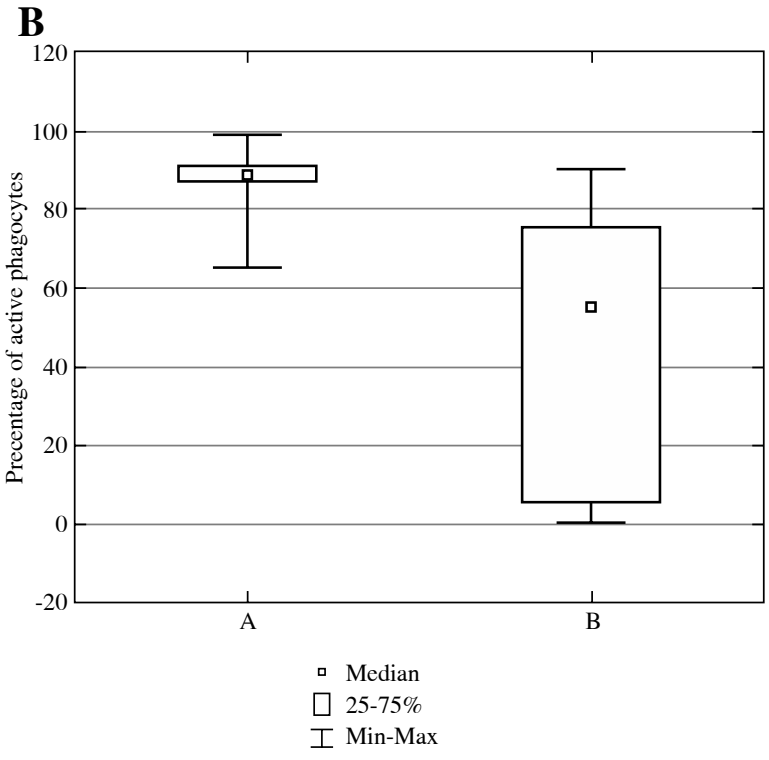

Fig. 3B. The percentage of granulocytes that showed phagocytosis in polytrauma patients on the $3^{\text {rd }}$ day when compared to healthy controls $(p<0.05)$

D

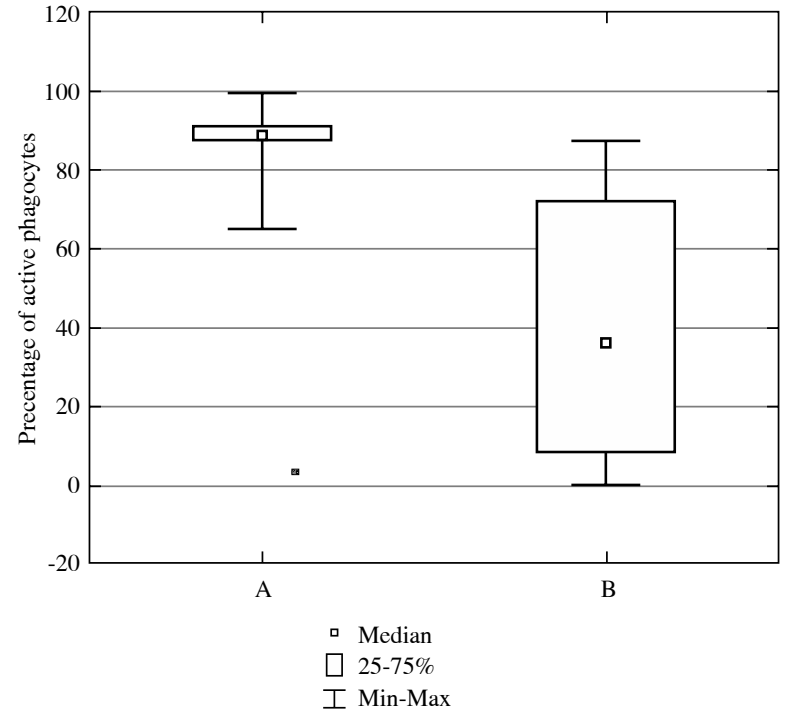

Fig. 3D. The percentage of granulocytes that showed phagocytosis in polytrauma patients on the $6^{\text {th }}$ day when compared to healthy controls $(p<0.05)$

The oxidative burst in circulating granulocytes depends on the underlying insult. The measurement of the degree of the oxidative burst in circulating granulocytes is a predictive factor used to distinguish between survivors and non-survivors. This measurement is completed on the $3^{\text {rd }}$ day after admission to the ICU [14]. 


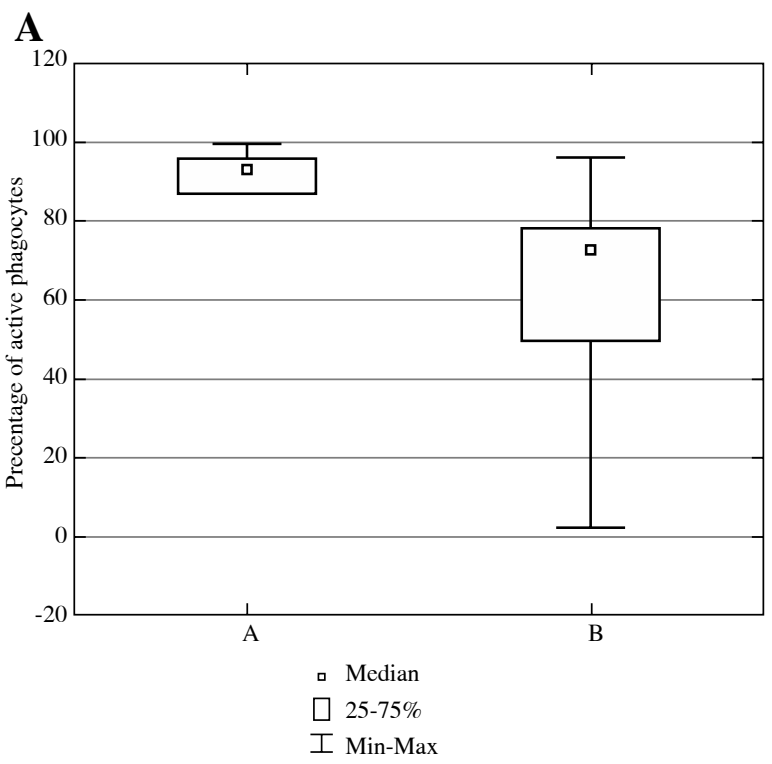

Fig. 4A. The percentage of granulocytes that showed enzymatic activity with reactive oxygen metabolites production in polytrauma patients on the $3^{\text {rd }}$ day when compared to the CNS-injured patients $(p<0.05)$

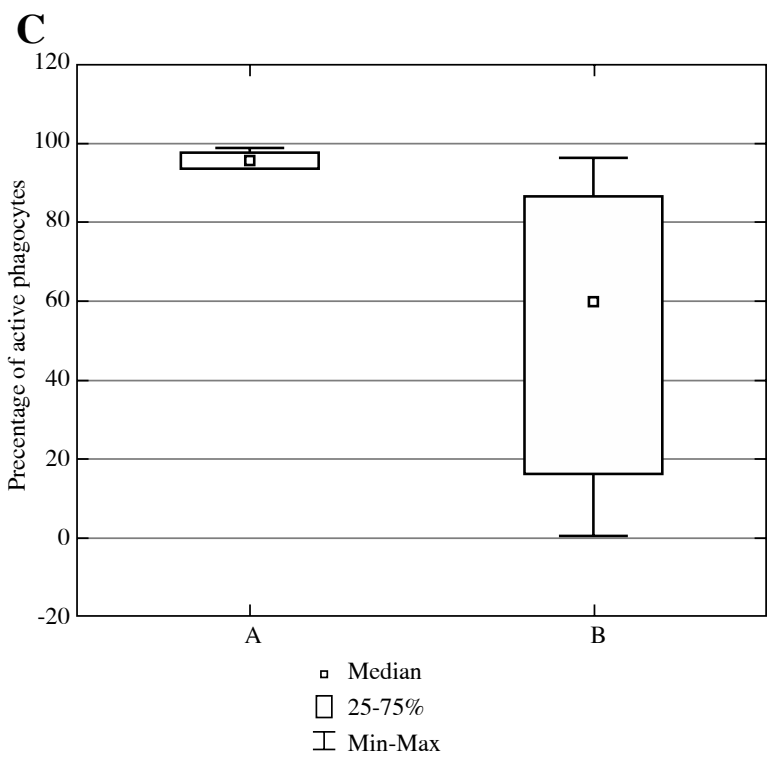

Fig. 4C. The percentage of granulocytes that showed enzymatic activity with reactive oxygen metabolites production in polytrauma patients on the $6^{\text {th }}$ day when compared to CNS-injured patients $(p<0.05)$

In our study, during the observation period, between the $3^{\text {rd }}$ and $6^{\text {th }}$ day after polytrauma injury, most of the measured parameters were statistically significantly changed. According to the literature, the recovery of the phagocytic arm would probably happen after the $6^{\text {th }}$ day post injury. However, we can observe the tendency (with no statistical
B

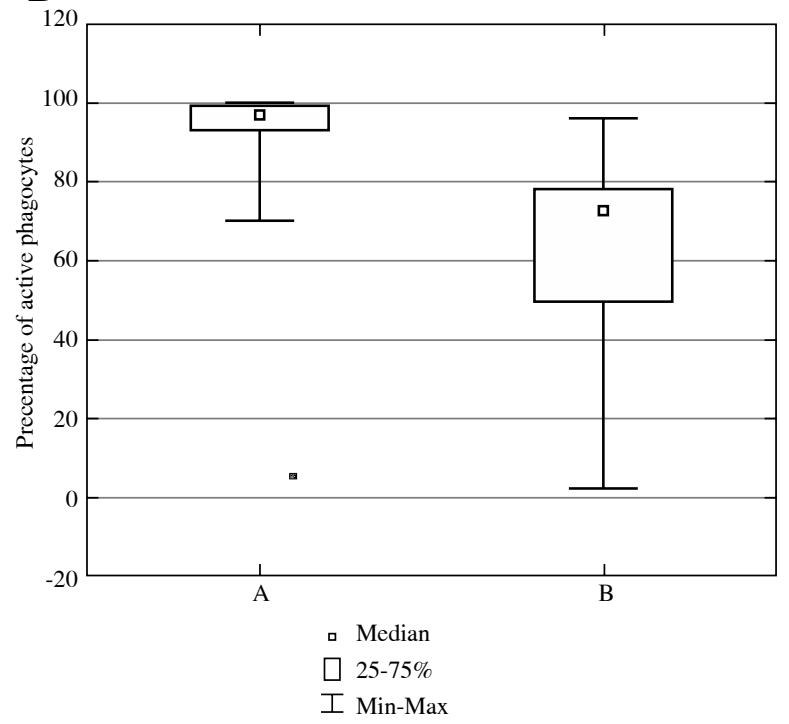

Fig. 4B. The percentage of granulocytes that showed enzymatic activity with reactive oxygen metabolites production in polytrauma patients on the $3^{\text {rd }}$ day when compared to healthy controls $(p<0.05)$

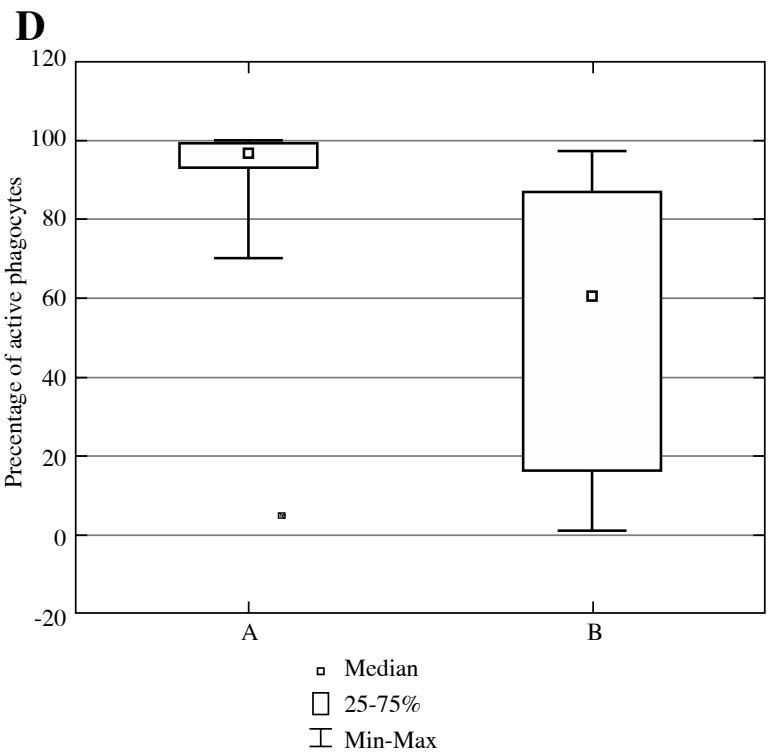

Fig. 4D. The percentage of granulocytes that showed enzymatic activity with reactive oxygen metabolites production in polytrauma patients on the $6^{\text {th }}$ day when compared to healthy controls $(p<0.05)$

significance) in a decreasing percentage of cells taking part in phagocytosis and enzymatic activity (reactive oxygen metabolites production).

The decreased percentage of cells taking part in phagocytosis and enzymatic activity and, at the same time, higher intracellular enzymatic activity in polytrauma patients 


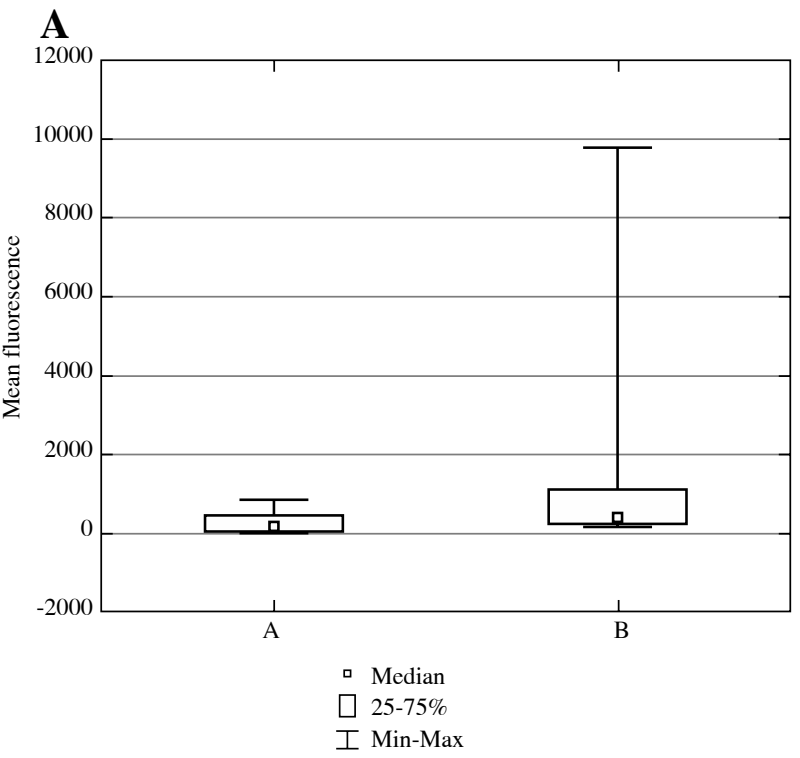

Fig. 5A. Enzymatic activity (associated with reactive oxygen metabolites production) in polytrauma on the $3^{\text {rd }}$ day in polytrauma patients when compared to healthy controls $(p<0.05)$

comparing to controls and the isolated (CNS) injury group could be explained by the process of granulocyte depletion during immunostimulation after trauma [23].

Multiple organ failure (MOF) and infections are important contributors to the morbidity and mortality associated with the polytrauma injury. Multiple organ failure is very likely to develop (could be triggered) as a result of definitive surgery performed at the time when there is a phagocytic arm deficiency.

The change in the functional behavior of the peripheral blood granulocytes could be an independent risk factor that could trigger the "second-hit" injury and infection [15]. During the phases of hyperinflammation and immunosuppression, severely injured patients are highly susceptible to sustain the so-called second-hit insults. Therefore, the ideal timing for the definitive surgery is somewhere between these two immunological phases. The empirically assigned time-window is about 5-10 days after trauma [6]. According to our results, this "time-window" is rather earlier or a couple of weeks later.

It seems that polytrauma-injured patients are at a risk of further immunological disturbances and are at a high risk of infection during the observed period of time. We know what the pattern of inflammatory response after trauma looks like: it starts with SIRS (could be followed by early MODS) and then develops into CARS (could be followed by late MODS) [25]. The decision-making process should be individualized in accordance to the clinical status of the patient, and the more exacerbating procedures should be performed at a later date.

It is well known that a decrease in the oxidative burst of granulocytes is associated with bacterial or fungal in-

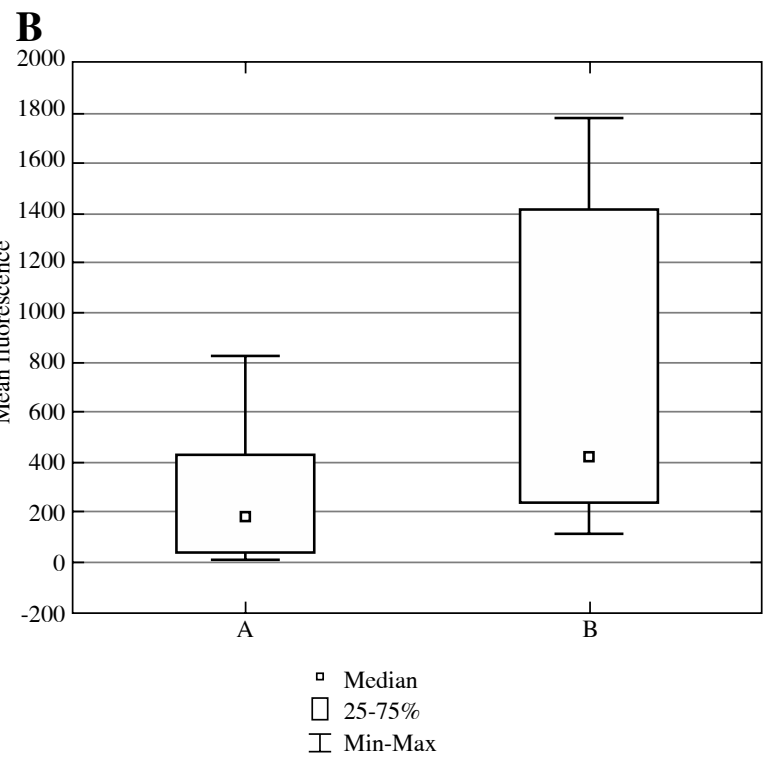

Fig. 5B. Enzymatic activity (associated with reactive oxygen metabolites production) in polytrauma on the $6^{\text {th }}$ day in polytrauma patients when compared to healthy controls $(p<0.05)$

fections [16]. The higher incidence of an early-onset pneumonia was found in patients with neurotrauma [17, 18]. However, infections are not only specific to CNS-injured patients, but also common to all patients treated in the ICU. Especially, in patients with a prolonged intubation since such intubation increases the risk of lung infection. Infections that start on the $3^{\text {rd }}$ day after injury are presumed to be nosocomial [19].

We have to take into consideration the fact that patients with a relatively low GCS who are most likely to be ventilated and sedated, as a result of which they would have reduced mobility, receive IV fluids, and have altered calorific intake. All of the above constitute multiple cofactors that might influence the results and cannot be excluded.

The clinical and immunological parameters related to pneumonia were revised and compared, yielding a result that the levels of chosen cytokines (IL-6 and IL-10) could not confirm the diagnosis of pneumonia earlier than the clinical parameters [20].

Surgical techniques adjusted to trauma are crucial to limit the pathologic systemic response. For the patient who is in a vulnerable phase of immunological defense it is rather important to have an individually adjusted surgical approach, as well as to control the immune response in order to avoid the "second-hit" insult and infections [21].

\section{Conclusions}

In a vulnerable phase when the patient's immunological defense is hypoactive and uncontrolled, the techniques and timing used in trauma surgery should be appropriately adjusted to limit the systemic response. An individually 
adjusted surgical approach and immune monitoring are important in order to avoid the "second-hit" injuries.

\section{The authors declare no conflict of interest.}

\section{References}

1. Baue AE, Durham R, Faist E (1998): Systemic inflammatory response syndrome (SIRS), multiple organ dysfunction syndrome (MODS), multiple organ failure (MOF): are we winning the battle? Shock 10: 79-89.

2. Keel M, Trentz O (2005): Pathophysiology of polytrauma. Injury 36: 323-341.

3. Kossman T, Hans VH, Imhof HG (1995): Intrathecal and serum IL-6 and the acute-phase respose in patients with severe traumatic brain injuries. Shock 4: 311-317.

4. Meisel Ch, Schwab JM, Prass K, et al. (2005): Central Nervous System injury-induced immune deficiency syndrome. Nature Reviews Neuroscience 6: 775-786.

5. Wolach B, Sazbon L, Gavrieli R, et al. (1993): Some aspects of the humor al and neutrophil functions In post comatose awareness patients. Brain Inj 7: 401-410.

6. Stahel PF, Smith WR, Moore EE (2007): Role of biological modifiers regulating the immune response after trauma injury. Injury 38: 1409-1422.

7. Meixensberger J, Roosen K (1998): Clinical and pathophysiological significance of severe neurotrauma in polytraumatized patients. Langenbacke's Arch Surg 383: 214-219.

8. Botha AJ, Moore FA, Moore EE, et al. (1997): Base deficit after major trauma directly relates to neutrophil CD11b expression: a proposed mechanism of shock-induced organ injury. Int Care Med 23: 504-509.

9. Sawyer DW, Donowitz GR, Mandell GL (1989): Polymorphonuclear neutrophils: An effective antimicrobial force. Rev Infect Dis 11 Suppl 7: S1532-S1544.

10. Bender MM, Gross CE, Howard DB (1997): Neutrophil activation in acute human central nervous system injury. Neurol Res 19: 588-592.

11. Hoyt DB, Ozkan AN, Hansbrough JF, et al. (1990): Head injury: an immunologic deficit in T-cell activation. J Trauma 30: 759-766.

12. Miller CH, Quattrocchi KB, Frank EH, et al. (1991): Humoral and cellular immunity following severe head trauma: review and current investigations. Neurol Res 13: 117-124.

13. Wolach B, Baehner RL, Boxer LA (1982): Review: clinical and laboratory approach to the management of neutrophil dysfunction. Isr J Med Sci 18: 897-916.

14. McCallion K, McAuley D, Harkin D, et al. (2003): Phagocyte priming as a prognostic indicator in the intensive care unit. J Trauma 55: 1089-1094.

16. Townsend RN, Lheureau T, Protetch J, et al. (1998): Timing fracture repair in patients with severe brain injury (Glasgow Coma Scale score <9). J Trauma 44: 977-983.

17. Babior BM (1984): The respiratory burst of phagocytes. J Clin Invest 73: 599-601.

18. Helling TS, Evans LL, Fowler DL, et al. (1988): Infectious complications in patients with severe head injury. J Trauma 28: 1575-1577.

19. Berrouane Y, Daudenthun I, Riegel B, et al. (1988): Early onset pneumonia in neurosurgical intensive care unite patients. J Hosp Infect 40: 275-280.
20. Dziedzic T, Słowik A, Szczudlik A (2004): Nosocomial infections and immunity: lessons from brain-injured patients. Crit Care 8: 266-270.

21. Andermahr J, Greb A, Hensler T, et al. (2002): Pneumonia in multiple injured patients: a prospective controlled trial on early prediction using clinical and immunological parameters. Inflamm Res 51: 265-272.

22. Gebhart F, Huber-Lang M (2008): Polytrauma-pathophysiology and management principles. Langenbecks Arch Surg 393: 825-831.

23. Brown KA, Brain SD, Pearson JD, et al. (2006): Neutrophils in development of multiple organ failure in sepsis. Lancet 368: 157-169.

24. Bumabasirevic V, Karamarcovic A, Lesic A, Bumbasirevic M (2005): Trauma related sepsis and multiple organ failure: current concepts in the diagnosis and management. Current Orthopaedics 19: 314-321.

25. Mannick JA, Rodrick ML, Lederer JA (2001): The immunologic response to injury. J Am Coll Surg 193: 237-244. 